\title{
Comparison of the effect of different media on the clinical outcomes of the density-gradient centrifugation/swim-up and swim-up methods
}

\author{
Eun-Kyung Kim ${ }^{1}$, Eun-Ha Kim¹, Eun-Ah Kim ${ }^{1}$, Kyung-Ah Lee ${ }^{2}$, Ji-Eun Shin ${ }^{1,3}$, Hwang Kwon ${ }^{1,3}$ \\ ${ }^{1}$ Fertility Center, CHA Bundang Medical Center, Seongnam; ${ }^{2}$ Department of Biomedical Science, College of Life Science, Seongnam; ${ }^{3}$ Department of \\ Obstetrics and Gynecology, CHA University College of Medicine, Seongnam, Korea
}

Objective: Sperm must be properly prepared in in vitro fertilization (IVF)-embryo transfer (ET) programs in order to control the fertilization rate and ensure that embryos are of high quality and have appropriate developmental abilities. The objective of this study was to determine the most optimal sperm preparation method for IVF.

Methods: Patients less than 40 years of age who participated in a fresh IVF-ET cycle from November 2012 to March 2013 were included in this study. Poor responders with less than three mature oocytes were excluded. Ham's F-10 medium or sperm-washing medium (SWM) was used in combination with the density-gradient centrifugation/swim-up (DGC-SUP) or SUP methods for sperm preparation. A total of 429 fresh IVF-ET cycles were grouped according to the media and methods used for sperm preparation and retrospectively analyzed (DGC-SUP/Ham's F-10, $\mathrm{n}=82$; DGC-SUP/SWM, $\mathrm{n}=43$; SUP/Ham's F-10, $\mathrm{n}=181$; SUP/SWM, $\mathrm{n}=123$ ).

Results: There were no significant differences among these four groups with respect to the mean age of the female partners, duration of infertility, number of previous IVF cycles, and retrieved oocytes. We determined that both the DGC-SUP and SUP methods for sperm preparation from whole semen, using either Ham's F-10 or SWM media, result in comparable clinical outcomes, including fertilization and pregnancy rates. Conclusion: We suggest that both media and both methods for sperm preparation can be used for selecting high-quality sperm for assistive reproductive technology programs.

Keywords: Assisted reproductive technology; Density gradient centrifugation; Intra cytoplasmic sperm injection; In vitro fertilization; Swim-up

\section{Introduction}

Human cervical mucus is known to select viable spermatozoa in the due course of normal and healthy pregnancies, acting as a natu-

Received: Dec 30, 2014 · Revised: Feb 25, 2015 · Accepted: Mar 7, 2015 Corresponding author: Hwang Kwon

Fertility Center, CHA Bundang Medical Center, 59 Yatap-ro, Bundang-gu, Seongnam 463-712, Korea

Tel: +82-31-780-5871 Fax:+82-31-780-5881 E-mail: hkwon@cha.ac.kr

*Part of this work was presented at the 46th Annual Meeting of the American Society for Reproductive Medicine held in Boston, MA, USA, October 12-17, 2013.

*This research was supported by LG Life Sciences (BD2013-039).

This is an Open Access article distributed under the terms of the Creative Commons Attribution Non-Commercial License (http://creativecommons.org/licenses/by-nc/3.0/) which permits unrestricted non-commercial use, distribution, and reproduction in any medium, provided the original work is properly cited. ral barrier against non-viable spermatozoa. However, this process of natural selection is bypassed in assisted reproductive technology (ART) programs. Consequently, it is very important to develop highquality procedures for the removal of seminal plasma in order to prepare and select of normal, healthy sperm for ART treatment.

Human ejaculate is composed of several components in addition to sperm, such as seminal liquid, epithelial cells, immature and necrotic sperm cells, and blood cells. Bacteria are also found in some patient samples. These components are involved in the production of toxic or bioactive substances such as decapacitation factors and free oxygen radicals that may impair the fertilization rate of the egg $[1,2]$. For this reason, removing the sperm from seminal fluid is a very important step in ART procedures [3]. More recently, it has been reported that fertilization with unselected apoptotic spermatozoa may contribute to failures in ART cycles [4]. Sperm DNA fragmentation is in- 
creased in poor-quality semen samples and is correlated with failed fertilization, impaired preimplantation development, and less frequent pregnancy outcomes $[5,6]$. An effective sperm separation method is therefore required for ART treatments to be successful.

Many methods have been developed for separating human spermatozoa from seminal plasma, including the swim-up (SUP) method, self-migration sedimentation, glass wool filtration, magnetic-activated cell sorting (MACS), and the use of a density gradient medium [4]. Among these methods, the methods that are most commonly applied in ART therapy are SUP and discontinuous density gradient centrifugation (DGC) using silane-coated colloidal silica particles in suspension.

It also has been shown that diluting the semen sample with a medium can improve sperm function and enhance the chances of fertilization. It has been suggested that media composed of HEPES, bicarbonate, calcium, and magnesium may enhance the chances of successful fertilization [7-12]. Both the complex Ham's F-10 medium and a simple medium composed of modified human tubal fluid are currently used for washing human sperm in clinical settings $[13,14]$. Some researchers have suggested that the use of these media results in higher-quality sperm, and improved ART outcomes have been reported with the use of either sperm selection or dilution. Recently, HEPES-buffered modified human tubal fluid has been developed and is commercially available as sperm washing medium (SWM).

No study has yet reported data comparing the clinical outcomes that result from the use of Ham's F-10 medium and SWM. Therefore, in this study, we studied the effects of these two different media in combination with the density-gradient centrifugation/swim-up (DGC-SUP) and the SUP sperm preparation methods, in order to determine the best SWM and processing methods for ART treatment by comparing clinical outcomes, with a focus on IVF pregnancy rates and associated metrics.

\section{Methods}

\section{Experimental design, patients and inclusion criteria}

We retrospectively evaluated 429 cycles performed from November 2012 to March 2013. The inclusion criteria were as follows: regular menstrual cycles of 25-35 days, baseline follicle-stimulating hormone levels $<15 \mathrm{IU} / \mathrm{L}$, anti-Müllerian hormone levels $\geq 1.0 \mathrm{ng} / \mathrm{mL}$, the presence of both ovaries and an intact uterus, and the absence of polycystic ovaries. Couples were excluded from this study if the female partner was over 40 years old or had poor ovarian function (less than three matured oocytes in a stimulation cycle), and if the male partner had undergone testicular sperm extraction or had a low total sperm count $\left(<5 \times 10^{6} / \mathrm{mL}\right)$. No patient had received any hormone therapy for at least 60 days preceding the study.
We assigned the couples into four groups according to the media and techniques used for sperm preparation: DGC-SUP with Ham's F-10 medium (group 1, $n=82$ ), DGC-SUP with SWM (group 2, $n=43$ ), SUP with Ham's F-10 medium (group $3, n=181$ ) and SUP with SWM (group $4, n=123$ ). The couples' semen samples were evaluated according to the World Health Organization's 1999 criteria for whether in vitro fertilization (IVF) is indicated, and intracytoplasmic sperm injection (ICSI) was performed in couples who had low sperm concentration, motility, or quality, or who had experienced low or failed fertilization in previous IVF cycles.

\section{Semen sample collection and preparation}

All semen samples were collected by masturbation after two to seven days of ejaculatory abstinence. After liquefaction for 20-30 minutes at room temperature, the semen samples were evaluated. The decision of performing DGC-SUP or SUP was made based on the quality of the semen sample. DGC-SUP was performed when the semen sample had low motility ( $<50 \%$ motility count), high viscosity with elevated leukocytes (over $2 \times 10^{6} / \mathrm{mL}$; LeucoScreen, Bioscreen, Torrance, CA, USA), or a high level of debris. All other semen samples were prepared using the SUP method.

\section{DGC/SUP method}

Discontinuous DGC was performed using a sterile pipette. The lower layer ( $90 \%$ Isolate, Irvine Scientific, Santa Ana, CA, USA) was first transferred into a conical centrifuge tube. Using a new sterile pipette, the upper layer (50\% Isolate, Irvine Scientific) was gently dispensed on top of the lower layer. A liquefied $2.0 \mathrm{~mL}$ semen sample was then placed on top of the upper layer and the tube was centrifuged for 10 minutes at $300 \mathrm{~g}$. This process was repeated using additional tubes until the entire sample of ejaculate was processed. The upper and lower layers were carefully aspirated without disturbing the pellet and discarded. Using a transfer pipette, $4.0 \mathrm{~mL}$ of Ham's F-10 medium (Irvine Scientific) with $0.5 \%$ synthetic serum substitute (Irvine Scientific) or $4.0 \mathrm{~mL}$ of HEPES-buffered SWM (Irvine Scientific) was added onto the pellet and the resuspended pellet was centrifuged for five minutes at $250 \mathrm{~g}$. The washing procedure was repeated twice. The supernatant was then removed and the pellet was suspended in 0.5 $\mathrm{mL}$ of Ham's F-10 medium or SWM, which was gently layered on top of the pellet, and the tube was inclined at an angle of 45 degrees and incubated at $35^{\circ} \mathrm{C}$ for at least $30-40$ minutes. After the incubation period, a sterile pipette was used to aspirate the supernatant and transfer it to a sterile $5-\mathrm{mL}$ conical tube. Sperm count and motility were determined in the recovered fractions (Figure 1).

\section{SUP method}

In groups 3 and 4, the semen sample was washed in a 15-mL Corn- 


\section{Total: 429 cycles}

20-30 min of room temperature liquefaction

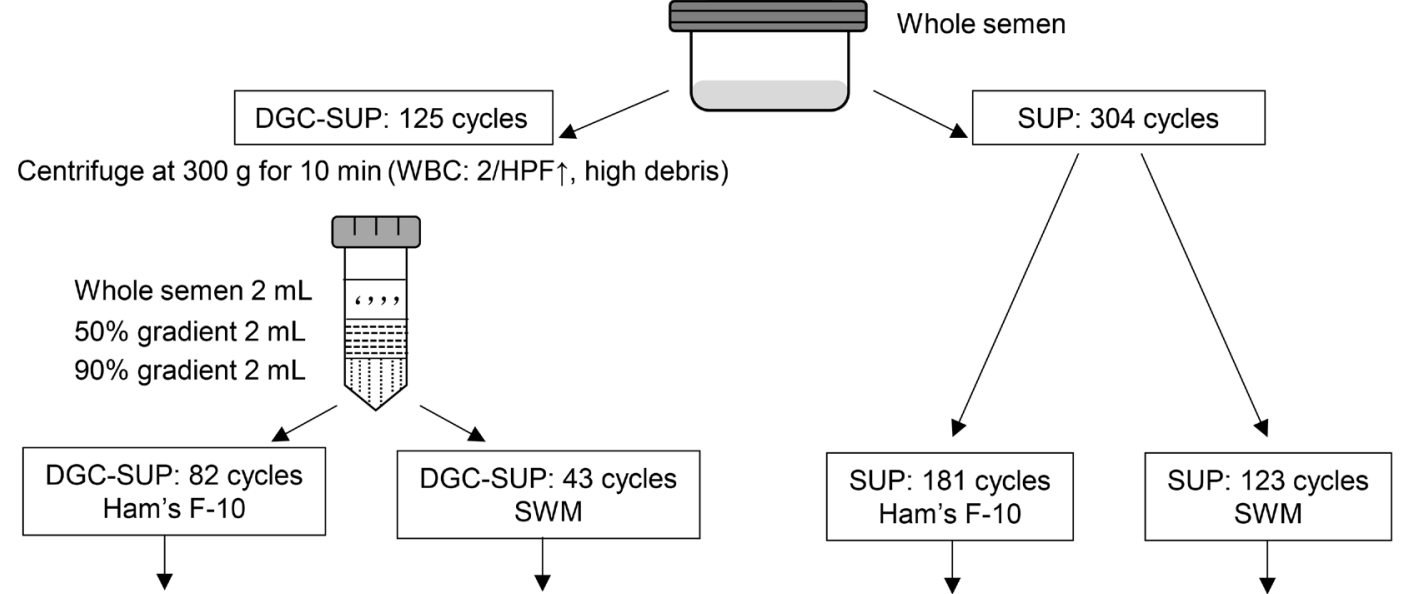

Centrifuge at $250 \mathrm{~g}$ for $5 \mathrm{~min}$, repeat wash twice

Centrifuge at $250 \mathrm{~g}$ for $5 \mathrm{~min}$, repeat wash twice

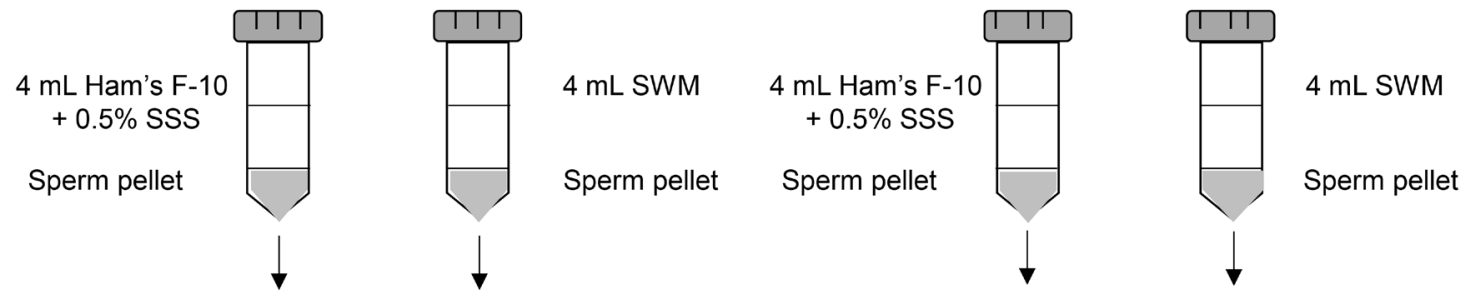

Incubate at $35^{\circ} \mathrm{C}$ for $30-40$ min at a $45^{\circ}$ angle

Incubate at $35^{\circ} \mathrm{C}$ for $30-40 \mathrm{~min}$ at a $45^{\circ}$ angle
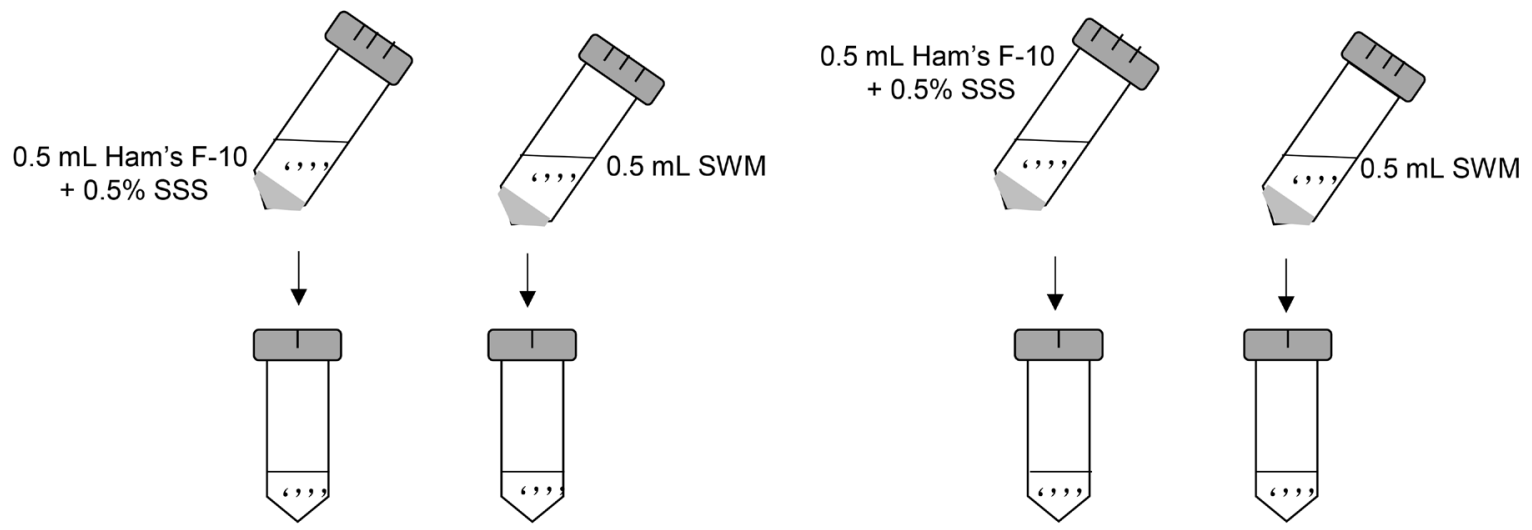

Recovered spermatozoa

$0.3 \mathrm{~mL} \quad 5-6 \times 10^{6} / \mathrm{mL}$

Figure 1. Schematic diagram for preparing sperm with the density gradient centrifugation-swim-up (DGC-SUP) and swim-up (SUP) methods using Ham's F-10 medium and sperm washing medium (SWM). WBC, white blood cell; HPF, high power field; SSS, synthetic serum substitute.

ing tube (Corning incorporated Life Science, New York, NY, USA) with $4 \mathrm{~mL}$ of Ham's F-10 medium supplemented with $0.5 \%$ synthetic serum substitute, or with $4 \mathrm{~mL}$ of SWM, and centrifuged at $250 \mathrm{~g}$ for five minutes. The washing procedure was repeated twice. The supernatant was discarded and $0.5 \mathrm{~mL}$ of medium was gently layered on top of the sperm pellet to allow motile sperm to swim up. The tube was then inclined at an angle of 45 degrees and incubated at $35^{\circ} \mathrm{C}$ for at least 30-40 minutes. After the incubation period, a sterile pipette was used to aspirate the supernatant and transfer it to a sterile 5-mL conical tube. Sperm count and motility were estimated. 


\section{Medium}

SWM is a modified form of human tubal fluid, and Ham's F-10 medium is supplemented with proteins. The composition of each medium used in this study is summarized in Table 1.

\section{Ovarian stimulation, oocyte retrieval, and embryo transfer}

Patients were subjected to ovarian stimulation with the use of gonadotropins (follicle-stimulating hormone or human menopausal gonadotropin) and gonadotropin-releasing hormone analogues or antagonists were used for controlled ovarian hyperstimulation. Patients received human chorionic gonadotropin when the diameter of the two or three leading follicles was $>18 \mathrm{~mm}$. Ultrasound-guided oocyte retrieval was performed 36 hours after the human chorionic gonadotropin injection, and luteal support consisting of vaginally administered progesterone was provided.

The insemination procedure performed on the oocytes was conventional IVF, ICSI, or split IVF-ICSI. We decided on ICSI or split IVF-ICSI to minimize or eliminate fertilization failure in couples who were in their first cycle or for whom one year had elapsed after IVF (Table 2).
Approximately 16-20 hours after the procedure, fertilization was confirmed by the presence of two pronuclei and the extrusion of the second polar body. The embryos were cultured in $2 \mathrm{~mL}$ of culture medium (Quinn's Advantage Cleavage Medium, SAGE, Trumbull, CT, USA) supplemented with $20 \%$ synthetic protein substitute (SAGE, Trumbull, $\mathrm{CT}, \mathrm{USA}$ ) in a humidified atmosphere with $6 \% \mathrm{CO}_{2}$ at $37^{\circ} \mathrm{C}$ for three days. Embryo transfer was performed on day three of devel-

Table 2. Number of cycles performed using each of the different fertilization procedures

\begin{tabular}{lcccccc}
\hline \multirow{2}{*}{ Methods (media) } & \multicolumn{2}{c}{ DGC-SUP } & & \multicolumn{2}{c}{ SUP } \\
\cline { 2 - 3 } \cline { 6 - 7 } \cline { 6 - 7 } & Ham's F-10 & SWM & & Ham's F-10 & SWM \\
\hline No. of cycles & 82 & 43 & & 181 & 123 \\
Conventional IVF (cycles) & 36 & 20 & & 8 & 6 \\
ICSI (cycles) & 13 & 4 & & 112 & 71 \\
Split IVF-ICSI (cycles) & 33 & 19 & & 61 & 46 \\
\hline
\end{tabular}

DGC-SUP, density gradient centrifugation/swim-up; SUP, swim-up; SWM, sperm washing medium; IVF, in vitro fertilization; ICSI, intra cytoplasmic sperm injection.

Table 1. Composition of Ham's F-10 medium and sperm washing medium

\begin{tabular}{|c|c|c|}
\hline \multicolumn{2}{|c|}{ Ham's F-10 } & \multirow[t]{2}{*}{ SWM } \\
\hline Sodium chloride & Potassium chloride & \\
\hline Glucose & L-Aspartic acid & Sodium chloride \\
\hline L-Asparagine $\cdot \mathrm{H}_{2} \mathrm{O}$ & L-Glutamine & Human serum albumin \\
\hline L-Glutamic acid & L-Isoleucine & HEPES free acid \\
\hline L-Histidine $\mathrm{HCl} \cdot \mathrm{H}_{2} \mathrm{O}$ & L-Methionine & Sodium bicarbonate \\
\hline L-Lysine $\mathrm{HCl}$ & L-Serine & Glucose \\
\hline L-Proline & L-Tyrosine $2 \mathrm{Na} \cdot 2 \mathrm{H}_{2} \mathrm{O}$ & Potassium chloride \\
\hline L-Tryptophan & Phenol red, Na salt & Calcium chloride \\
\hline Folic acid & d-Biotin & Sodium phosphate monobasic \\
\hline Vitamin B-12 & Pyridoxine $\mathrm{HCl}$ & HEPES sodium salt \\
\hline Pantothenic acid, Ca salt & Thiamine $\mathrm{HCl}$ & Magnesium sulfate \\
\hline Riboflavin & Cupric sulfate $\mathrm{CuSO}_{4} \cdot 2 \mathrm{H}_{2} \mathrm{O}$ & Sodium pyruvate \\
\hline Thimidine & Riboflavin & SSR (Synthetic serum substitute) \\
\hline Nicotinic acid amide & Thimidine & Gentamicin sulfate \\
\hline Thioctic acid, dihydrochloride & Zinc sulfate $\mathrm{ZnSO}_{4} \cdot 7 \mathrm{H}_{2} \mathrm{O}$ & Phenol red \\
\hline Ferrous sulfate $\mathrm{FeSO}_{4} \cdot 7 \mathrm{H}_{2} \mathrm{O}$ & Calcium chloride, anhydrous & \\
\hline Sodium phosphate, dibasic $\mathrm{Na}_{2} \mathrm{HPO}_{4}$ anhydrous & Sodium bicarbonate & \\
\hline Chloline chloride & Inositol & \\
\hline Potassium phosphate (monobasic $\mathrm{KH}_{2} \mathrm{PO}_{4}$ ) & Nocitinic acid amide & \\
\hline L-Arginine $\mathrm{HCl}$ & Thioctic acid, dihydrochloride & \\
\hline L-Cysteine $\mathrm{HCl} \cdot \mathrm{H}_{2} \mathrm{O}$ & Ferrous sulfate $\mathrm{FeSO}_{4} \cdot 7 \mathrm{H}_{2} \mathrm{O}$ & \\
\hline Glycine & Thiamine $\mathrm{HCl}$ & \\
\hline L-Leucine & Cupric sulfate $\mathrm{CuSO}_{4} \cdot 5 \mathrm{H}_{2} \mathrm{O}$ & \\
\hline L-Phenylalanine & Pyruvic acid, Na salt & \\
\hline L-Threonine & Magnesium sulfate, $\mathrm{MgSO}_{4}$, anhydrous & \\
\hline L-Valine & L-Glutamine (200 mM) & \\
\hline Hypoxanthine, 2Na salt & & \\
\hline
\end{tabular}

HEPES, 4-(2-hydroxyethyl)-1-piperazineethanesulfonic acid; SWM, sperm washing medium; SSR, synthetic serum substitute. 
opment, using a soft catheter with transabdominal ultrasound guidance. One to three embryos were transferred per patient. A pregnancy test was performed 13 days after embryo transfer. All women with a positive test underwent a transvaginal ultrasound scan two weeks after the positive test.

\section{Statistical analysis}

The data were expressed as mean \pm standard deviation. The stu-

Table 3. Comparison of semen parameters in the four groups

\begin{tabular}{lcclcc}
\hline \multirow{2}{*}{ Parameters } & \multicolumn{2}{c}{ DGC-SUP } & & \multicolumn{2}{c}{ SUP } \\
\cline { 2 - 3 } \cline { 5 - 6 } \cline { 5 - 6 } & Ham's F-10 & SWM & & Ham's F-10 & SWM \\
\hline Strict morphology (\%) & $11.4 \pm 4.4$ & $12.1 \pm 4.6$ & & $10.5 \pm 4.8$ & $11.7 \pm 4.1$ \\
Initial & & & & & \\
$\quad$ Volume $(\mathrm{mL})$ & $3.2 \pm 1.4$ & $3.4 \pm 1.3$ & & $3.3 \pm 1.4$ & $3.6 \pm 1.4$ \\
Count $\left(\times 10^{6} / \mathrm{mL}\right)$ & $113.8 \pm 72.2$ & $98.9 \pm 59.9$ & & $92.7 \pm 57.9$ & $82.9 \pm 47.6$ \\
$\quad$ Motility $(\%)$ & $43.9 \pm 14.2$ & $41.8 \pm 15.5$ & & $46.3 \pm 14.3$ & $46.6 \pm 13.3$ \\
Final & & & & & \\
Volume $(\mathrm{mL})$ & 0.3 & 0.3 & & 0.3 & 0.3 \\
Count $\left(\times 10^{6} / \mathrm{mL}\right)$ & $5.4 \pm 1.6$ & $5.6 \pm 1.2$ & & $6.4 \pm 2.3$ & $6.2 \pm 2.2$ \\
Motility $(\%)$ & 100 & 100 & & $99.5 \pm 3.0$ & $97.1 \pm 12.1$ \\
\hline
\end{tabular}

Values are presented as mean \pm standard deviation.

DGC-SUP, density gradient centrifugation/swim-up; SUP, swim-up; SWM, sperm washing medium; Strict morphology was carried out according to the 1999 World Health Organization criteria. dent's $t$-test was used. A $p$-value $<0.05$ was considered to indicate statistical significance.

\section{Results}

The sperm parameters in the four groups are summarized in Table 3. The initial progressive motility of the sperm before treatment was lower in the DGC-SUP groups than in the SUP groups, but there were no differences in the morphology (assessed using the strict morphology approach), initial volume, final volume, and count.

Patient characteristics such as mean age, infertility duration, number of previous IVF cycles, fertilization, embryo transfer, and pregnancy rates are summarized in Table 4. No differences among the four groups were observed regarding the age of the male and female partner, the duration of infertility, and the number of previous IVF cycles. There was no significant difference in the female partner's basal levels of follicle-stimulating hormone and anti-Müllerian hormone among groups (data not shown). Regarding the causes of infertility, 207 of 429 patients (48.3\%) had unexplained fertility, 127 (29.6\%) had infertility with a complex cause associated with the female partner, 62 (14.5\%) had tubal factor infertility and 39 (9\%) had male factor infertility. The patients with male factor infertility had lower than normal sperm counts, but did not suffer from conditions that would have caused them to meet the exclusion criteria outlined in the

Table 4. Comparison of clinical outcomes in the four groups

\begin{tabular}{|c|c|c|c|c|}
\hline \multirow{2}{*}{ Methods (media) } & \multicolumn{2}{|c|}{ DGC-SUP } & \multicolumn{2}{|c|}{ SUP } \\
\hline & Ham's F-10 & SWM & Ham's F-10 & SWM \\
\hline No. of cycles & 82 & 43 & 181 & 123 \\
\hline Female age (yr) & $33.5 \pm 3.2$ & $34.1 \pm 3.3$ & $33.7 \pm 3.3$ & $34.1 \pm 3.1$ \\
\hline Male age (yr) & $35.8 \pm 3.6$ & $35.9 \pm 3.7$ & $35.9 \pm 3.4$ & $35.8 \pm 3.8$ \\
\hline Infertility duration (yr) & $3.7 \pm 1.9$ & $4.8 \pm 2.1$ & $4.8 \pm 2.5$ & $4.5 \pm 2.8$ \\
\hline No. of previous IVF cycles & $1.4 \pm 0.6$ & $1.3 \pm 0.6$ & $1.3 \pm 0.6$ & $1.4 \pm 0.7$ \\
\hline No. of retrieved oocytes (mean \pm standard deviation) & $1,010(12.3 \pm 5.7)$ & $499(11.6 \pm 5.9)$ & $2,184(12.1 \pm 5.8)$ & $1,499(12.2 \pm 6.3)$ \\
\hline No. of inseminated or injected oocytes & 966 & 483 & 1,988 & 1,382 \\
\hline No. of 2PN after conventional IVF (\%) & $439 / 626(70.1)$ & $208 / 310(67.1)$ & $342 / 501(68.3)$ & $333 / 467(71.3)$ \\
\hline No. of 2PN after ICSI (\%) & $281 / 340(82.7)$ & $140 / 173(80.9)$ & $1,197 / 1,487(80.5)$ & $736 / 915(80.4)$ \\
\hline No. of fertilizations (\%) ${ }^{\mathrm{a})}$ & $720 / 966(74.5)$ & $348 / 483(72.1)$ & 1,539/1,988 (77.4) & $1,069 / 1,382(77.4)$ \\
\hline No. of transferred embryos & $2.9 \pm 0.6$ & $3.0 \pm 0.6$ & $2.9 \pm 0.8$ & $2.9 \pm 0.7$ \\
\hline Pregnancies (\%) & $36 / 82(43.9)$ & $21 / 43(48.8)$ & $89 / 181(49.2)$ & $57 / 123(46.3)$ \\
\hline Chemical pregnancies (\%) & $1(2.8)$ & $1(4.8)$ & $8(9.0)$ & $5(8.8)$ \\
\hline Abortions (\%) & $4(11.1)$ & $5(23.8)$ & $12(13.5)$ & $6(10.5)$ \\
\hline Ectopic pregnancies (\%) & $1(2.8)$ & $2(9.5)$ & $3(3.4)$ & $6(10.5)$ \\
\hline Delivery rates (\%) & $25(69.4)$ & $9(42.9)$ & $53(59.6)$ & $29(50.9)$ \\
\hline Patients lost to follow-up (\%) & $5(13.9)$ & $4(19.0)$ & $13(14.6)$ & $11(19.3)$ \\
\hline
\end{tabular}

Values are presented as mean \pm standard deviation or number (\%).

DGC-SUP, density gradient centrifugation/swim-up; SUP, swim-up; SWM, sperm washing medium; IVF, in vitro fertilization; 2PN, two pronuclei; ICSI, intra cytoplasmic sperm injection.

a)Fertilization rate: number of 2PN instances. 
methods. Again, no significant differences were found among the four groups.

The clinical outcomes were not significantly different between the DGC-SUP and SUP groups with regard to all parameters, including the number of retrieved oocytes and the fertilization rates of both conventional IVF and ICSI treatment. The four groups also had a similar number and frequency of embryos that were transferred on day three. No clinical results exhibited a statistically significant difference among the four groups.

The presence of similar clinical outcomes among the four groups suggest that the four methods of preparing sperm that we studied led to comparable fertilization rates, as well as embryos of comparable quality and developmental potential.

\section{Discussion}

Immediately after ejaculation, sperm are not able to participate in fertilization. Instead, they obtain this ability while passing through the female reproductive organs. Significant advances have recently been made in understanding the molecular mechanisms involved in certain aspects of capacitation and the acrosome reaction associated with the egg-sperm fusion process [7]. Moreover, abnormal sperm parameters such as extremely low sperm concentration, poor sperm motility, and abnormal morphology contribute to low fertility [15].

Spermatozoa are protected by various materials in the seminal plasma itself. However, materials such as seminal liquid, epithelial cells, immature and necrotic sperm cells, red blood cells, white blood cells, and even bacteria can produce toxic or bioactive substances, such as decapacitation factors or free oxygen radicals that can impair the capacity of the sperm to fertilize the egg. For this reason, washing the sperm is necessary to improve their function.

Separation of the sperm from the whole semen is an important and necessary step. Various semen preparation techniques have been developed in ART programs to isolate motile, morphologically normal, and healthy spermatozoa for use in IVF. A number of sperm washing techniques can be used to separate motile sperm from nonmotile or dead sperm as well as other contaminating cell debris. The DGC-SUP and SUP methods are two of the most widely used techniques in semen preparation [16]. The most commonly used methods are the simple washing of spermatozoa, which is based on selfmigration of the spermatozoa, and the SUP and DGC-SUP methods, in which preparations with different density gradients are used. SUP from a washed pellet is easy to perform and cost-effective. It is the oldest and most commonly used sperm separation method, and is therefore used in many IVF laboratories around the world [17]. However, the DGC-SUP method improves success rates in intrauterine insemination cycles [18], and has an increased ability to concentrate normal sperm [16] in comparison with the SUP method. It has been reported that the DGC-SUP method has comparable results to the electrophoretic method for sperm recovery, motility, and DNA fragmentation [19].

The presence of DNA damage within spermatozoa is negative associated with fertilization, cleavage, blastulation, implantation, and pregnancy $[15,20]$. Xue et al. [21] reported that DGC-SUP led to the enrichment of sperm with normal morphology and intact DNA in teratozoospermic patients. The effect of sperm DNA fragmentation on pregnancy was not affected by the processing technique used to separate the sperm from the whole semen sample or the fertilization procedure, as measured both before and after SUP. It is difficult to determine how sperm DNA fragmentation affects clinical outcomes; while the DNA integrity of sperm can be evaluated, the repair capacity of oocytes remains an enigma [21-23]. Stevanato et al. [24] have suggested that semen processing by DGC-SUP is not generally useful in selecting sperm with higher double-strand DNA integrity. However, both DGC-SUP and SUP methods allow a healthy sperm population with a low percentage of apoptotic sperm to be obtained [25]. In addition, Borges et al. [26] reported that both the DGC-SUP and SUP methods were useful in recovering sperm with improved characteristics, and they obtained similar IMSI outcomes using these two methods. In this study, the sample number of DGC-SUP groups was smaller than the SUP groups, because the DGC-SUP method was used to treat semen samples with $<50 \%$ motility, highly elevated leukocytes, or high levels of debris. Nonetheless, we could not find any significant differences in the clinical results of the four sperm preparation techniques we used.

Various materials exist to enhance the competence of sperm. In particular, bicarbonate in seminal plasma stimulates adenylate cyclase in spermatozoa, and the bicarbonate-sensitive adenylate cyclase system has been shown to regulate sperm motility [10]. Calcium is an essential bivalent cation for the acrosome reaction, and extracellular calcium is also required for the sperm capacitation process [11]. Proteins and magnesium are also responsible for chromatin stability in sperm, motility, and the acrosome reaction [27,28]. Murase et al. [9] reported that albumin and HEPES are beneficial for the longterm storage of boar sperm. In this experiment, we compared the use of a complex culture medium that was traditionally used in early ART programs (Ham's F-10 medium), with the use of a simple medium that has recently been used in IVF programs for semen washing (SWM). HEPES is specifically contained in SWM. However, in this study, we did not find any difference in clinical outcomes among the four different combinations of the two media and the two SUP methods. Therefore, our results suggest that flexibility is appropriate in the choice of a medium or method for sperm preparation in ART programs, and we propose that there is no need to use expensive IVF 
culture media such as SWM.

\section{Conflict of interest}

No potential conflict of interest relevant to this article was reported.

\section{References}

1. Alvarez JG, Sharma RK, Ollero M, Saleh RA, Lopez MC, Thomas AJ $\mathrm{Jr}$, et al. Increased DNA damage in sperm from leukocytospermic semen samples as determined by the sperm chromatin structure assay. Fertil Steril 2002;78:319-29.

2. Lanzafame FM, La Vignera S, Vicari E, Calogero AE. Oxidative stress and medical antioxidant treatment in male infertility. Reprod Biomed Online 2009;19:638-59.

3. Lampiao F, du Plessis SS. Comparing the Multi-ZSC one-step standardized swim-up method to the double-wash swim-up method with regard to the effects of sperm separation on morphology, head morphometry, and acrosome reaction inducibility. Fertil Steril 2006;86:739-41.

4. Bucar S, Goncalves A, Rocha E, Barros A, Sousa M, Sa R. DNA fragmentation in human sperm after magnetic-activated cell sorting. J Assist Reprod Genet 2015;32:147-54.

5. Borini A, Tarozzi N, Bizzaro D, Bonu MA, Fava L, Flamigni C, et al. Sperm DNA fragmentation: paternal effect on early post-implantation embryo development in ART. Hum Reprod 2006;21:287681.

6. Bungum $M$, Humaidan $P$, Axmon A, Spano $M$, Bungum $L$, Erenpreiss J, et al. Sperm DNA integrity assessment in prediction of assisted reproduction technology outcome. Hum Reprod 2007; 22:174-9.

7. Gadella BM, Van Gestel RA. Bicarbonate and its role in mammalian sperm function. Anim Reprod Sci 2004;82-83:307-19.

8. Harrison RA, Gadella BM. Bicarbonate-induced membrane processing in sperm capacitation. Theriogenology 2005;63:342-51.

9. Murase T, Imaeda N, Yamada H, Takasu M, Taguchi K, Katoh T. Dilution of boar ejaculates with BTS containing HEPES in place of bicarbonate immediately after ejaculation can reduce the increased inducibility of the acrosome reaction by treatment with calcium and calcium ionophore A23187, which is potentially associated with boar subfertility. J Reprod Dev 2010;56:309-14.

10. Okamura N, Tajima Y, Soejima A, Masuda H, Sugita Y. Sodium bicarbonate in seminal plasma stimulates the motility of mammalian spermatozoa through direct activation of adenylate cyclase. J Biol Chem 1985;260:9699-705.

11. Witte TS, Schafer-Somi S. Involvement of cholesterol, calcium and progesterone in the induction of capacitation and acrosome re- action of mammalian spermatozoa. Anim Reprod Sci 2007;102: 181-93.

12. Zollner U, Zollner KP, Dietl J, Steck T. Semen sample collection in medium enhances the implantation rate following ICSI in patients with severe oligoasthenoteratozoospermia. Hum Reprod 2001;16:1110-4.

13. Yenilmez E, Yildirmis S, Yulug E, Aydin S, Tekelioglu Y, Erdem E, et al. Ham's F-10 medium and Ham's F-10 medium plus vitamin $E$ have protective effect against oxidative stress in human semen. Urology 2006;67:384-7.

14. Quinn P, Lydic ML, Ho M, Bastuba M, Hendee F, Brody SA. Confirmation of the beneficial effects of brief coincubation of gametes in human in vitro fertilization. Fertil Steril 1998;69:399-402.

15. Larson-Cook KL, Brannian JD, Hansen KA, Kasperson KM, Aamold ET, Evenson DP. Relationship between the outcomes of assisted reproductive techniques and sperm DNA fragmentation as measured by the sperm chromatin structure assay. Fertil Steril 2003; 80:895-902.

16. Prakash P, Leykin L, Chen Z, Toth T, Sayegh R, Schiff I, et al. Preparation by differential gradient centrifugation is better than swimup in selecting sperm with normal morphology (strict criteria). Fertil Steril 1998;69:722-6.

17. Henkel RR, Schill WB. Sperm preparation for ART. Reprod Biol Endocrinol 2003;1:108.

18. Karamahmutoglu H, Erdem A, Erdem M, Mutlu MF, Bozkurt N, Oktem $M$, et al. The gradient technique improves success rates in intrauterine insemination cycles of unexplained subfertile couples when compared to swim up technique; a prospective randomized study. J Assist Reprod Genet 2014;31:1139-45.

19. Fleming SD, llad RS, Griffin AM, WuY, Ong KJ, Smith HC, et al. Prospective controlled trial of an electrophoretic method of sperm preparation for assisted reproduction: comparison with density gradient centrifugation. Hum Reprod 2008;23:2646-51.

20. Benchaib M, Braun V, Lornage J, Hadj S, Salle B, Lejeune H, et al. Sperm DNA fragmentation decreases the pregnancy rate in an assisted reproductive technique. Hum Reprod 2003;18:1023-8.

21. Xue X, Wang WS, Shi JZ, Zhang SL, Zhao WQ, Shi WH, et al. Efficacy of swim-up versus density gradient centrifugation in improving sperm deformity rate and DNA fragmentation index in semen samples from teratozoospermic patients. J Assist Reprod Genet 2014;31:1161-6.

22. Meseguer M, Santiso R, Garrido N, Garcia-Herrero S, Remohi J, Fernandez JL. Effect of sperm DNA fragmentation on pregnancy outcome depends on oocyte quality. Fertil Steril 2011;95:124-8.

23. Sakkas D, Alvarez JG. Sperm DNA fragmentation: mechanisms of origin, impact on reproductive outcome, and analysis. Fertil Steril 2010;93:1027-36. 
24. Stevanato J, Bertolla RP, Barradas V, Spaine DM, Cedenho AP, Ortiz V. Semen processing by density gradient centrifugation does not improve sperm apoptotic deoxyribonucleic acid fragmentation rates. Fertil Steril 2008;90:889-90.

25. Ricci G, Perticarari S, Boscolo R, Montico M, Guaschino S, Presani G. Semen preparation methods and sperm apoptosis: swim-up versus gradient-density centrifugation technique. Fertil Steril 2009; 91:632-8.

26. Borges E Jr, Setti AS, Vingris L, Figueira Rde C, Braga DP, laconelli A Jr. Intracytoplasmic morphologically selected sperm injection outcomes: the role of sperm preparation techniques. J Assist Reprod Genet 2013;30:849-54.

27. Rogers BJ, Yanagimachi R. Competitive effect of magnesium on the calcium-dependent acrosome reaction in guinea pig spermatozoa. Biol Reprod 1976;15:614-9.

28. Bahmanpour S, Namavar MR, Talaei-Khozani T, Mazaheri Z. The effect of the follicular fluid on sperm chromatin quality in comparison with conventional media. Eur Rev Med Pharmacol Sci 2012;16:1840-6. 